\title{
Preparation of Catalysts based on Iron(III) Porphyrins Heterogenized on Silica obtained by the Sol-Gel Process for Hydroxylation and Epoxidation Reactions
}

\author{
Kelly A. D. F. Castro, ${ }^{a}$ Matilte Halma, ${ }^{a}$ Guilherme S. Machado, ${ }^{a}$ Gustavo P. Ricci, ${ }^{b}$ \\ Geani M. Ucoski, ${ }^{a}$ Katia J. Ciuffi ${ }^{b}$ and Shirley Nakagaki ${ }^{*, a}$ \\ ${ }^{a}$ Universidade Federal do Paraná, Departamento de Química - Laboratório de Química \\ Bioinorgânica e Catálise, CP 19081, 81531-990 Curitiba-PR, Brazil \\ ${ }^{b}$ Universidade de Franca, Departamento de Química, CP 82, 14404-600, Franca-SP, Brazil
}

\begin{abstract}
Catalisadores sólidos foram preparados pela interação de ferroporfirinas com sílica obtida pelo processo sol-gel. A presença da ferroporfirina na matriz de sílica e a morfologia das partículas obtidas foram estudadas por espectroscopia eletrônica na região do ultravioleta e visível, difratometria de raios $\mathrm{X}$ de pó, espectroscopia vibracional na região do infravermelho, microscopia eletrônica de transmissão, ressonância paramagnética eletrônica e análises termogravimétricas. A atividade catalítica dos sólidos obtidos foi investigada frente a oxidação dos substratos $(Z)$ cicloocteno, cicloexeno e cicloexano por iodosilbenzeno em meio de diclorometano e acetonitrila. Os resultados catalíticos obtidos foram comparáveis àqueles observados quando as ferroporfirinas em solução (catálise homogênea) foram utilizadas.
\end{abstract}

Solid catalysts have been prepared by chemical interaction of iron(III) porphyrins with the surface of the pores of a silica matrix obtained by the sol-gel method. The presence of the complexes in the silica matrix and the morphology of the obtained particles were studied by UV-Vis spectroscopy, powder X-ray diffractometry, infrared spectroscopy, transmission electron microscopy, electron paramagnetic resonance and thermogravimetric analysis. The catalytic activity of the immobilized iron(III) porphyrins in the oxidation of (Z)-cyclooctene, cyclohexene and cyclohexane was evaluated in dichloromethane/acetonitrile 1:1 solvent mixture (v/v) using iodosylbenzene as oxidant. Results were compared with those achieved with the homogeneous counterparts.

Keywords: iron porphyrin, silica, sol-gel, oxidation, cytochrome P-450 mimetic systems

\section{Introduction}

Porphyrins and related macrocycles provide an extremely versatile synthetic base for a variety of applications in the area of new materials. ${ }^{1-3}$ Metalloporphyrins are important examples of macrocyclic complexes ${ }^{4}$ that have demonstrated catalytic ability in a wide variety of reactions over the last 30 years. Such reactions include oxidative processes, especially in homogeneous media.

More recently, porous metalloporphyrin networks have proven to be potentially applicable as efficient heterogeneous catalysts ${ }^{5}$ after immobilization in organic amorphous polymers, amorphous inorganic matrices, or crystalline inorganic materials such as silica, ${ }^{6,7}$ zeolites, ${ }^{8,9}$ clay from the smectite group (montmorillonite), ${ }^{10-12}$ layered

\footnotetext{
*e-mail: shirley@quimica.ufpr.br
}

double hydroxides, ${ }^{13,14}$ tubular and fibrous matrices, ${ }^{15}$ silica matrix obtained by the sol-gel process, among others. ${ }^{16-23}$ This versatile class of molecules is efficient and selective for the heterogeneous catalytic hydroxylation and epoxidation of a wide variety of organic substrates.

Catalyst immobilization enables site-isolation of the metal center and is one of the approaches to reducing metalloporphyrin degradation because it may prevent molecular aggregation or bimolecular self-destruction reactions, ${ }^{20}$ which all lead to deactivation of the catalytically active metalloporphyrin species. ${ }^{19,21}$ Indeed, catalyst stability is essential for recycling procedures and recovery of valuable metalloporphyrins..$^{23,24}$

To obtain an ideal heterogeneous catalytic system consisting of porphyrin-matrix, the porphyrin active site must be readily accessible for both charge transfer and mass transport. Therefore, a study on the best possible approach 
to building heterogeneous catalysts based on porphyrins is crucial to obtain economically viable applications for these systems.

Although various works in the literature have reported the heterogenization of metalloporphyrin catalysts, we have verified that much has to be done in terms of optimization and matrix preparation. The investigation of factors such as particle shape and size, as well as active site distribution on the matrices, may furnish catalysts with potentially applicable features in a wide variety of fields in both the scientific and industrial areas.

Stöber et $a l .{ }^{25}$ have described a method for the preparation of monodispersed spherical silica particles of nanometric dimensions through tetraethylorthosilicate (TEOS) hydrolysis in ethanol, employing basic conditions and ambient temperature. This method is widely applied nowadays because its simplicity enables the production of spherical particles of different sizes. ${ }^{21}$ Thus, this can be an alternative process in the search for more efficient matrices for catalyst immobilization.

Our research team ${ }^{18,19}$ and others ${ }^{20,21}$ have observed that the addition of porphyrins to silica matrices obtained by the sol-gel methodology can affect particle porosity and morphology. As already reported, ${ }^{21}$ the presence of porphyrins can contribute to the polymerization of the silica network.

In the sol-gel methodology, control of the preparation conditions can easily alter the structure and properties of the obtained materials. The use of an acid catalyst can also be an attractive alternative approach in the preparation of the heterogeneous iron porphyrin catalysts. In the case of silica, the utilization of acidic catalysts leads to transparent gels of low porosity, whereas basic catalysts furnish translucent, highly porous gels. ${ }^{21}$
This work reports the immobilization of three structurally different iron porphyrins, namely: $\left.[\mathrm{Fe}(\mathrm{TMPyP})] \mathrm{Cl}_{5}\right]$, [5,10,15,20-tetrakis(4- $N$-methylpyridyl)porphyrin iron(III) chloride]; [Fe(TDFPP)]Cl], [5,10,15,20-tetrakis(2,6difluorophenyl)porphyrin iron(III) chloride] and [Fe(TDCPP)]Cl, [5,10,15,20-tetrakis(2,6-dichlorophenyl) porphyrin iron(III) chloride] (Figure 1) in the silica structure obtained by the hydrolytic sol-gel process. ${ }^{25}$ After characterization, the catalytic activity of the obtained solid compounds in oxidation reactions was investigated.

\section{Experimental}

\section{Materials}

All chemicals used in this study were purchased from Aldrich, Sigma or Merck and were of analytical grade. In particular, tetraethylorthosilicate (TEOS) was acquired from Aldrich; ethanol (EtOH), dichloromethane (dcm), dimethylformamide (dmf), methanol $(\mathrm{MeOH})$, isopropyl alcohol $\left(\mathrm{Pr}^{\mathrm{i}} \mathrm{OH}\right)$, and tetrahydrofuran (thf) were obtained from Merck. Iodosylbenzene (PhIO) was synthesized by hydrolysis of iodosylbenzenediacetate, ${ }^{26}$ and the obtained solid was carefully dried under reduced pressure and kept at $5^{\circ} \mathrm{C}$. $\mathrm{PhIO}$ purity was periodically controlled by iodometric titration. ${ }^{27}$ Deionized water was used in all experimental procedures.

Porphyrins: The free base porphyrins 5,10,15,20-tetrakis(1-methylpyridinium-4-yl)porphyrin $\left(\left[\mathrm{H}_{2}(\mathrm{TMPyP})\right]^{4+}\right), 5,10,15,20$-tetrakis(2,6-fluorophenyl) porphyrin $\left.\left(\left[\mathrm{H}_{2}(\mathrm{TDFPP})\right]\right)\right]$ and $5,10,15,20$-tetrakis $(2,6-$ dichlorophenyl)porphyrin ([ $\mathrm{H}_{2}$ (TDCPP)]) were synthesized, purified, and characterized following the previously described methodology. ${ }^{28-30}$

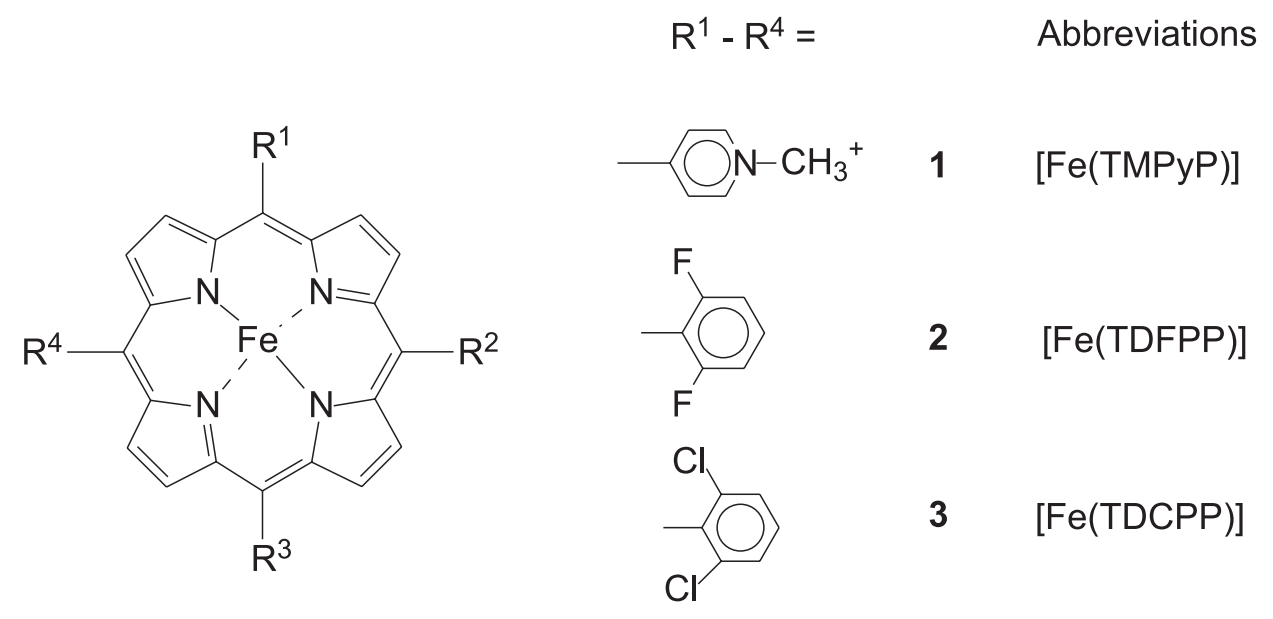

Figure 1. Schematic representation of the iron(III) porphyrins employed in this work. 
Iron(III) porphyrins: Iron(III) porphyrins (FePors) were obtained by metallation of the free base with ferrous chloride tetrahydrate in $\mathrm{dmf}$ following the method described by Adler et al. ${ }^{31,32}$ The FePors were purified by column chromatography on silica or alumina. The products were characterized by UV-Visible and EPR spectroscopy, and the data were consistent with the expected compound after the metallation reaction. [Fe(TMPyP)] (deionized water): $390 \mathrm{~nm}$ $\left(\varepsilon=13 \times 10^{3} \mathrm{~L} \mathrm{~mol}^{-1} \mathrm{~cm}^{-1}\right),[\mathrm{Fe}(\mathrm{TDFPP})]$ (dichlorometane) $412 \mathrm{~nm}\left(\varepsilon=45 \times 10^{3} \mathrm{~L} \mathrm{~mol}^{-1} \mathrm{~m}^{-1}\right),[\mathrm{Fe}(\mathrm{TDCPP})]$ (dichlorometane) $412 \mathrm{~nm}\left(\varepsilon=47 \times 10^{3} \mathrm{~L} \mathrm{~mol}^{-1} \mathrm{~cm}^{-1}\right)$, which were denoted FePor $\mathbf{1}$, FePor $\mathbf{2}$, and FePor $\mathbf{3}$, respectively. The positive charges and the chlorine counter ion will be omitted in the text for simplification purposes.

Immobilization of $\mathbf{1}, \mathbf{2}$, and $\mathbf{3}$ in silica prepared by the solgel process (FePor-SGB and FePor-SGA solids)

\section{Basic catalysis}

The FePor-SGB catalysts were prepared by the Stöber methodology, ${ }^{25}$ through hydrolysis of TEOS in ethanol/ methanol, employing $\mathrm{NH}_{4} \mathrm{OH}$ as catalyst, $1 \times 10^{-5} \mathrm{~mol}$ of the desired FePor, and the following molar ratios: $\mathrm{H}_{2} \mathrm{O} / \mathrm{Si}$ $(\mathrm{TEOS})=50, \mathrm{NH}_{3} / \mathrm{EtOH}=10$, and $\mathrm{H}_{2} \mathrm{O} / \mathrm{NH}_{3}=200 . \mathrm{An}$ alternative methodology utilizing isopropyl alcohol $\left(\mathrm{Pr}^{i} \mathrm{OH}\right)$ instead of ethanol $\left(\mathrm{NH}_{3} / \mathrm{Pr}^{i} \mathrm{OH}=10\right)$ in the presence of $7 \times 10^{-6}$ mol of FePor $\mathbf{1}, \mathbf{2}$ or $\mathbf{3}$ was also employed. The reaction mixture was kept at $40{ }^{\circ} \mathrm{C}$; the solution was stirred for $30 \mathrm{~min}$ and kept at about $60{ }^{\circ} \mathrm{C}$ for complete dryness. The final materials, gelatinized after about 14 days, consisted in transparent brown-emerald green stable gels. The wet gels were extensively washed with $\mathrm{MeOH}$, EtOH, $\mathrm{H}_{2} \mathrm{O}$ and dem by Soxhlet procedure, followed by drying at $70{ }^{\circ} \mathrm{C}$ for $24 \mathrm{~h}$. The FePor loading in the silica was quantified by UV-Vis spectroscopy, by measuring the amount of non-immobilized FePor recovered during the washing of the solid. A control sample containing no FePor was also synthesized in the same way as described above, furnishing a transparent colorless gel that was also washed with $\mathrm{MeOH}, \mathrm{EtOH}, \mathrm{H}_{2} \mathrm{O}$ and dem by the same Soxhlet procedure, and then dried in an oven at $70{ }^{\circ} \mathrm{C}$ for $24 \mathrm{~h}$.

\section{Acid catalysis}

The FePor-SGA catalyst was prepared by the sol-gel process through hydrolysis of TEOS in ethanol/methanol, employing $\mathrm{HCl}$ as catalyst, $1 \times 10^{-5} \mathrm{~mol}$ of the desired FePor and the following molar ratios: $\mathrm{H}_{2} \mathrm{O} / \mathrm{Si}($ TEOS $)=4$, thf $/ \mathrm{HCl}=308$, and $\mathrm{H}_{2} \mathrm{O} / \mathrm{HCl}=92 .{ }^{21}$ The reaction mixture was stirred for $1 \mathrm{~h}$ at room temperature, and kept at about
$70{ }^{\circ} \mathrm{C}$ for complete dryness. The final materials, gelatinized after about 7 days, consisted in transparent brown-emerald green stable gels similar to the FePor-SGB solids. The wet gels were also extensively washed with $\mathrm{MeOH}, \mathrm{EtOH}, \mathrm{H}_{2} \mathrm{O}$ and dcm by Soxhlet procedure, followed by drying for $24 \mathrm{~h}$ at $70{ }^{\circ} \mathrm{C}$. In the case of the acid sol-gel process, UV-Vis spectroscopy detected no FePor in the solvent recovered at the end of the washing process. A control sample containing no added FePor was also synthesized in the same conditions described above.

\section{Catalytic oxidation reaction}

Catalytic oxidation reactions were carried out in a $2 \mathrm{~mL}$ thermostatic glass reactor equipped with a magnetic stirrer placed inside a dark chamber. Alkene and alkane reactions were accomplished using FePor-SGB or FePor-SGA as catalysts (about $0.01 \mathrm{~g}$ ), and (Z)-cyclooctene, cyclohexene or cyclohexane as substrates. The alkenes had been previously purified on an alumina column. In a standard experiment, the solid catalyst and iodosylbenzene (FePor/ $\mathrm{PhIO}$ molar ratio $=1: 50$ ) were suspended in $0.400 \mathrm{~mL}$ of the solvent (dichloromethane/acetonitrile 1:1 mixture $\mathrm{v} / \mathrm{v}$ ) inside a $2 \mathrm{~mL}$ vial and degassed with argon for $15 \mathrm{~min}$. The substrate $($ FePor/substrate molar ratio $=1: 5000)$ was added, and the oxidation reaction was carried out for 1 to $24 \mathrm{~h}$, under magnetic stirring. Sodium sulfite was added at the end of the reaction, to eliminate the excess of iodosylbenzene. The soluble reaction products were separated from the solid catalyst by centrifugation and transferred to a volumetric flask. Then, the solid catalyst was washed several times with dichloromethane and acetonitrile, in order to extract any remaining product. The solution containing the final reaction solution plus the solvents from the washings of the solid catalyst was analyzed by gas chromatography. Product yields were quantified on the basis of PhIO, using $n$-octanol (acetonitrile solution, $1.0 \times 10^{-2} \mathrm{~mol} \mathrm{~L}^{-1}$ ) of high purity degree $(99.9 \%)$ as internal standard. Control reactions were carried out using the same procedure in the case of (a) the substrate alone, (b) substrate $+\mathrm{PhIO}$, and (c) substrate + $\mathrm{PhIO}+$ silica (without FePor). The FePor in solution was also tested as a catalyst (homogeneous catalysis), and the experimental procedure in this case was similar to that used for the heterogeneous catalysis.

After the first use, the solid catalyst was thoroughly washed and dried for reuse in another reaction, under the same experimental conditions employed in its first use.

\section{Characterization and apparatus}

Electronic spectra were recorded on an HP 8452A Diode Array UV-Vis spectrophotometer, in the $200-800 \mathrm{~nm}$ 
range. Spectra of the solid samples were recorded in a $0.1 \mathrm{~cm}$ path length quartz cell (Hellma) in nujol mull.

FTIR spectra were registered on a Biorad 3500 GX spectrophotometer in the 400 to $4000 \mathrm{~cm}^{-1}$ range, using $\mathrm{KBr}$ pellets. $\mathrm{KBr}$ was crushed with a small amount of the solid samples, and the spectra were collected with a resolution of $4 \mathrm{~cm}^{-1}$ and accumulation of 32 scans.

For the X-ray diffraction (XRD) measurements, selforiented films were placed on neutral glass sample holders. The measurements were performed in the reflection mode using a Shimadzu XRD-6000 diffractometer operating at $40 \mathrm{kV}$ and $40 \mathrm{~mA}\left(\mathrm{Cu}-\mathrm{K}_{\alpha}\right.$ radiation, $\left.\lambda=1.5418 \AA\right)$ with a dwell time of $1 \theta / \mathrm{min}$.

Electron paramagnetic resonance (EPR) measurements of the powder materials were carried out on an EPR BRUKER ESP 300E spectrometer (standard concavity: 4102-SP, frequency $\mathrm{X}$ band $9.5 \mathrm{GHz}$ ), at 293 or $77 \mathrm{~K}$, using liquid $\mathrm{N}_{2}$.

Thermogravimetric (TGA) measurements were performed on a Thermal Analyst TA Instrument SDT 600 Simultaneous DTA-TGA-DSC, in nitrogen, with a heating rate of $20^{\circ} \mathrm{C} \mathrm{min}-1$, from 25 to $1200^{\circ} \mathrm{C}$.

Transmission electron microscopy (TEM) was accomplished on an $80 \mathrm{kV}$ JEM 1200EXII Electron Microscope. A drop of a suspension of the catalyst powder was deposited on a 300 mesh copper grid (formvar/carbon).

Products from the catalytic oxidation reactions were identified using a Shimadzu GC-14B gas chromatograph (flame ionization detector) equipped with a DB-WAX capillary column, $30 \mathrm{~m}$ (J \& W Scientific).

Specific surface areas were determined by applying the BET method ${ }^{33}$ to the corresponding nitrogen adsorption isotherms, obtained by using a physical adsorption analyzer Micrometrics ASAP 2020. The samples were previously degassed by treatment at $180-200{ }^{\circ} \mathrm{C}$ until the system pressure reached $10 \mu \mathrm{mHg}$. The nitrogen adsorption data were obtained using $0.2 \mathrm{~g}$ of the sample.

\section{Results and Discussion}

The FePor-SGA and FePor-SGB solids were prepared through hydrolysis and condensation of the alkoxysilane (TEOS) in alcohol and water, using ammonia or $\mathrm{HCl}$ as catalysts in the presence of one of the FePors (1, 2 or $\mathbf{3})$. The hydrolysis and polycondensation reactions initiate at numerous sites as soon as TEOS and $\mathrm{H}_{2} \mathrm{O}$ are mixed. Under normal conditions, both hydrolysis and condensation may occur by acid or base-catalyzed bimolecular nucleophilic substitution reactions. The acid-catalyzed mechanisms proceed via rapid deprotonation of the $-\mathrm{OR}$ or $-\mathrm{OH}$ substituents bound to $\mathrm{Si},{ }^{21}$ whereas under basic conditions hydroxyl or silanolate anions attack Si directly. Hydrolysis of alkylsilicates followed by condensation in appropriate $\mathrm{pH}$ furnishes high purity silica particles. ${ }^{25}$ The choice between acid or basic catalysis for silica synthesis influences the nature of the prepared catalyst. For this reason, we decided to study the role played by $\mathrm{pH}$ in the catalytic activity of the materials prepared herein.

The control silica sample, containing no FePor, is white, whereas the solids obtained after FePor immobilization are of a light brown-green color that gradually deepens with increasing FePor concentration.

After the solids were dried, FePor loading in the silica was determined by measuring the difference between the absorbance of the complex in the solution prepared before the immobilization process and the absorbance of the solution and combined washings from the Soxhlet procedure obtained after FePor immobilization (Table 1).

Table 1. Immobilization of FePors in silicas obtained by the sol-gel process

\begin{tabular}{lccc}
\hline Solid & $\begin{array}{c}\text { Percentage of } \\
\text { immobilization }(\%)^{\mathrm{a}}\end{array}$ & \multicolumn{2}{c}{$\begin{array}{c}\text { Loading (concentration of } \\
\text { FePor in the silica) }\end{array}$} \\
\cline { 3 - 4 } & & $8.5 \times 10^{-6}$ & 0.83 \\
\hline FePor 1-SGA & 100 & $2.2 \times 10^{-5}$ & 2.1 \\
FePor 1-SGB & 98.0 & $9.0 \times 10^{-6}$ & 0.75 \\
FePor 2-SGA & 99.6 & $2.3 \times 10^{-5}$ & 1.9 \\
FePor 2-SGB & 95.4 & $8.4 \times 10^{-6}$ & 0.81 \\
FePor 3-SGA & 100 & $1.8 \times 10^{-5}$ & 1.7 \\
FePor 3-SGB & 96.7 &
\end{tabular}

${ }^{\text {a }}$ Iron porphyrin immobilized in the silica considering the initial mass used.

Table 1 reveals high FePor immobilization (> 95\%) in the silica support, and FePor immobilization is superior in the materials prepared by acid catalysis. The structure of the final silica materials explains the FePor percentage of immobilization. An open silica network arises during acid catalysis, which provides a larger number of available sites and facilitates FePor entrapment. In contrast, materials achieved by means of basic catalysis consist in interconnected silica spherical particles, thereby leading to lower FePor percentage of immobilization.

Acid catalysis and low $\mathrm{H}_{2} \mathrm{O} / \mathrm{Si}$ ratios furnish weakly branched polymeric sols and gels, which results in highly microporous xerogels with a very fine texture. Basic catalysis and high $\mathrm{H}_{2} \mathrm{O} / \mathrm{Si}$ ratios, on the other hand, produce colloidal particles that give rise to meso- or macroporous xerogels composed by translucent gels of high porosity. ${ }^{21,34}$

The mechanism through which FePor immobilization occurs in neutral medium is not completely understood. Interactions between the $\pi$-conjugated electron cloud 
of the macrocyclic rings and the structure of the highly hydroxylated silica are possible. Furthermore, the FePor can insert into the three-dimensional network of the silica resulting from $\mathrm{Si}-\mathrm{O}-\mathrm{Si}$ links. This leads to agglomeration of the molecules, thereby forming larger particles and agglomerates, or a colloidal sol (Figure 2).

In the case of FePor 1, in addition to the possible interactions mentioned above, electrostatic interactions between FePor $\mathbf{1}$ and the silica surface could also take place, giving rise to FePor immobilization on the surface of the support.

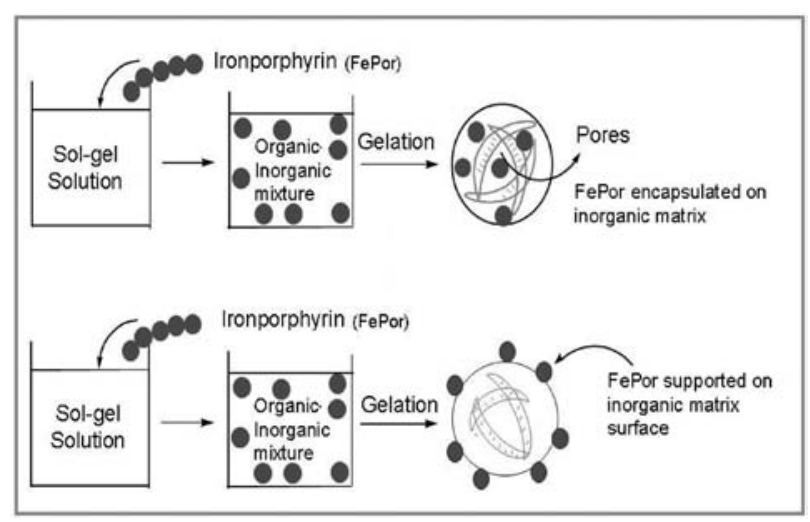

Figure 2. Schematic representation of the FePor-silica solid formed by the sol-gel process.

Figure 3 presents the transmission electron micrographs of the FePor-SG catalysts and the control materials (containing no FePor). As observed previously by us, ${ }^{19}$ addition of porphyrins to silica matrices obtained by the sol-gel methodology can affect particle morphology and agglomeration. Comparison of the micrographs obtained for the control silica SGB and FePor-SGB shows that the silica particles are more spherical in the presence of FePors. However, particle morphology is irregular in the cases of the FePor 2-SGB and FePor 3-SGB hybrid materials, but spherical for FePor 1-SGB.

The positive character of the charged FePor 1 may favor agglomeration of the FePor 1-SGB particles, which would account for the production of beads only in the presence of this FePor, under the experimental conditions of this work.

Wang et al..$^{23}$ observed that silica prepared by the Stöber ${ }^{25}$ methodology tends to absorb cationic porphyrins strongly. This is because this methodology can produce negatively charged spherical particles depending on the $\mathrm{pH}$ conditions employed during silica preparation. The silanol groups present on the material achieved at high $\mathrm{pH}$ can dissociate and produce negative charges, thus facilitating FePor adsorption. This is an excellent approach for the preparation of materials consisting of spheres. ${ }^{23}$

The solids obtained by immobilization of FePors 1, 2 and $\mathbf{3}$ in the silica achieved by the sol-gel process (acid and basic catalysis) as well as the control silica (without FePor) were analyzed by XRD (Figure not shown). The XRD patterns of all prepared solids revealed only a halo in the region of 20 and $30^{\circ}$ for $2 \theta$, which means that they are amorphous.

The presence of FePor in the hybrid FePor-SG solids was confirmed by EPR analysis. Figure 4 depicts the EPR spectra of SGA (a), FePor 1-SGA (b), FePor 1-SGB (c), FePor 2-SGA (d), FePor 2-SGB (e), FePor 3-SGA (f), and FePor 3-SGB (g) at $77 \mathrm{~K}$. The control silicas SGA (Figure 4a) and SGB (not shown) display EPR-silent spectra. The absence of EPR signals indicates that these solids are free of contaminating paramagnetic species that
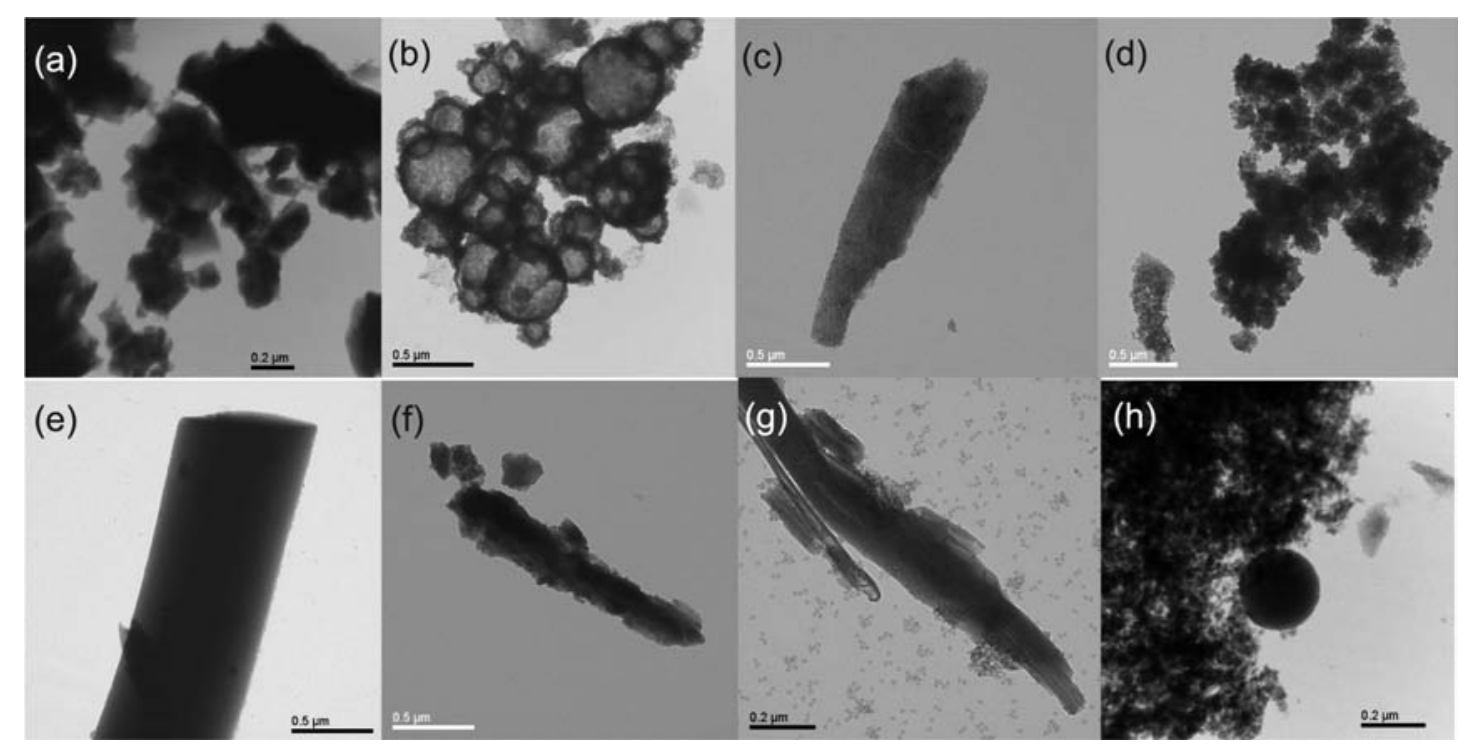

Figure 3. Transmission electron microscopy (TEM). (a) FePor 1-SGA; (b) FePor 1-SGB; (c) FePor 2-SGA; (d) FePor 2-SGB; (e) FePor 3-SGA; (f) FePor 3-SGB; (g) SGA-blank; (h) SGB-blank. 
could have been inserted into the silica during the synthetic process. All the prepared FePor-SG solids display EPR signals at $g=6.0$ (1000-1200 gauss), characteristic of high-spin iron(III) in axial symmetry, ${ }^{24,35-37}$ and a small signal at $g=4.3$, typical of high-spin iron(III) in rhombic symmetry. ${ }^{37,38}$ The latter EPR signal suggests that, upon immobilization, some or all FePor molecules may undergo a rhombic distortion because of their interaction with the structure of the support. Nevertheless, the axial symmetry signal at $g=6.0$ is still present in high intensity.

The presence of the typical EPR iron(III) signal in the silica solids confirms that during the immobilization process no significant demetallation of the FePors took place. Demetallation could have been caused by binding of the metal ion to the coordinating groups in the solid derivative, in the presence of the hydroxyl groups. ${ }^{37}$

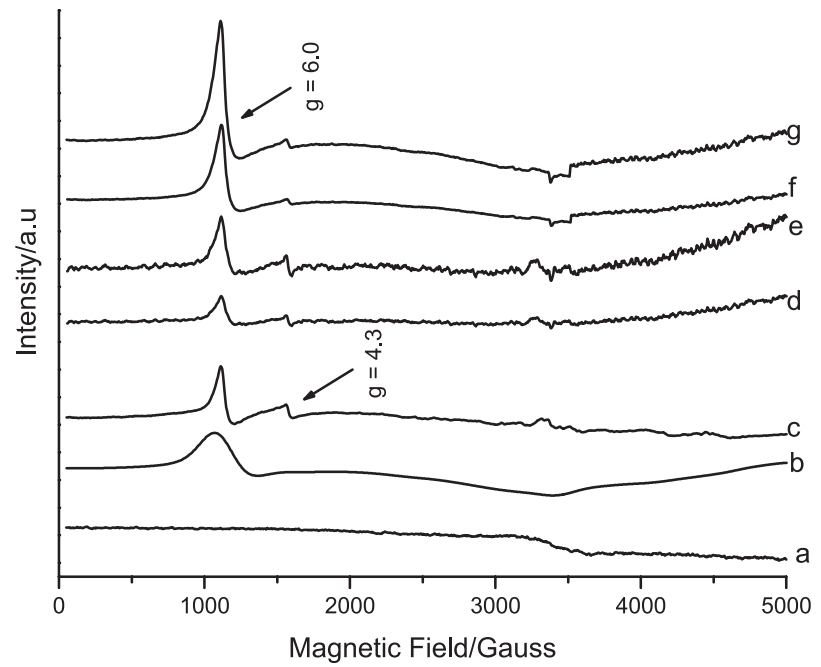

Figure 4. EPR spectra of solid samples obtained at 77K. (a) SGA; (b) FePor 1-SGA; (c) FePor 1-SGB; (d) FePor 2-SGA; (e) FePor 2-SGB; (f) FePor 3-SGA; (g) FePor 3-SGB.

Figure 5 shows the FTIR spectra of the solids FePor-SG prepared in this work (Figure 5a-5e).

The main characteristic of the FTIR spectrum of pure silica is an intense, wide band in the region of $3420 \mathrm{~cm}^{-1}$, due to the typical axial deformation of superficial $\mathrm{SiO}-\mathrm{H}$ groups. Also, there is a large band at $1630 \mathrm{~cm}^{-1}$, ascribed to water molecules adsorbed/absorbed in the silica. The band in the region of $1100 \mathrm{~cm}^{-1}$ is characteristic of the stretching of the 180-degree angle of the $\mathrm{Si}-\mathrm{O}-\mathrm{Si}$ groups of the four tetrahedral $\mathrm{SiO}_{2}$. The band at $960 \mathrm{~cm}^{-1}$ is assigned to $\mathrm{Si}-\mathrm{OH}$ vibration, and the band at $798 \mathrm{~cm}^{-1}$ is associated with the distorted structure of $\mathrm{SiO}_{4}{ }^{36}$ The FTIR bands of the FePors are not observed, probably because of the low concentration of the adsorbate compared with the support (Table 1). Nevertheless, some differences are noted when the spectrum of the control solid is compared with those of the FePor-SG materials. For instance, the characteristic $\mathrm{Si}-\mathrm{O}$ stretching band shifts to lower wavenumber after FePor immobilization. This shift is more pronounced for the FePor 1-SGA solid, indicating that this FePor interacts with the silica siloxane groups, and that FePor 1 might be located inside the matrix pores. ${ }^{39}$

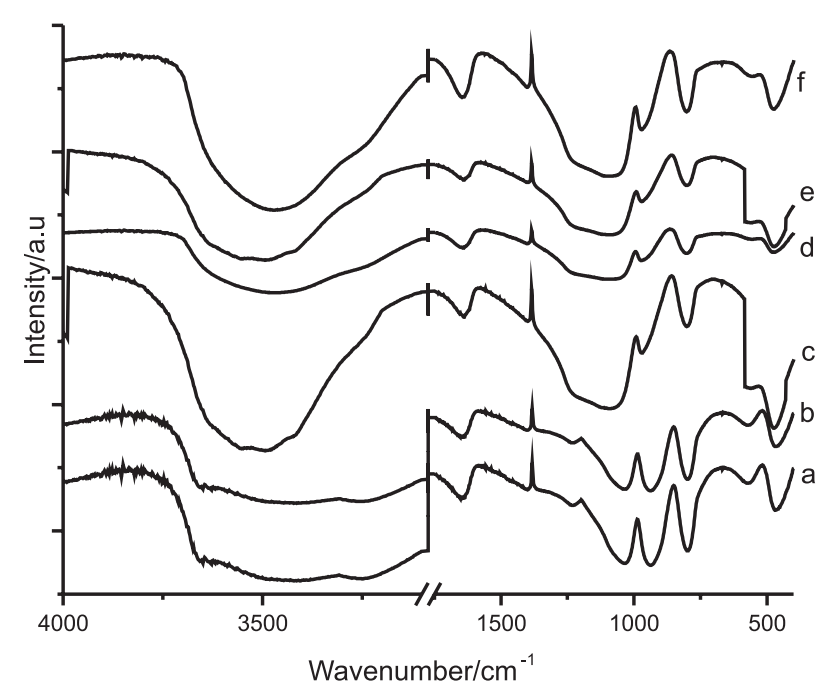

Figure 5. FTIR spectra (a) FePor 1-SGA; (b) FePor 1-SGB; (c) FePor 2-SGA; (d) FePor 2-SGB; (e) FePor 3-SGA; (f) FePor 3-SGB.

The prepared solids were also analyzed by UV-Vis spectroscopy in nujol mull (Figure 6). The spectra of all FePor-SG solids displayed a typical peak enlargement ${ }^{40}$ and presented the Soret band characteristic of FePors in the region of $400 \mathrm{~nm}$, thereby confirming the presence of FePor 1, 2, or $\mathbf{3}$ in the prepared silicas. However, the Soret band of all the FePor-SG materials was shifted to lower energy compared with the spectrum of the parent FePor in solution (spectrum collected in Nujol oil emulsion figure not shown). This demonstrates that no demetallation (characterized by a blue shift of the Soret band typical of the presence of a significant amount of free-base porphyrin $)^{18}$ or significant metal ion exchange between the metallocomplex and the support occurs during the immobilization process. On the other hand, the red-shifted Soret band obtained in the case of the immobilized FePors gives evidence that FePor immobilization in the solid causes no important steric constraints that would substantially modify the metalloporphyrin structure in the supported catalyst. ${ }^{36}$ In fact, the EPR spectrum (Figure 4) displays signals both at $g=6.0$ and $g=4.3$, thus confirming that only some distortion of the FePor takes place after the immobilization process. The UV-Vis spectrum of the solid does not allow for a more detailed analysis of the visible region. For metalloporphyrins in solution, this region often exhibits bands that aid understanding of the axial coordination of 
the metal and help determine whether dimeric species are formed during the immobilization process. ${ }^{41}$

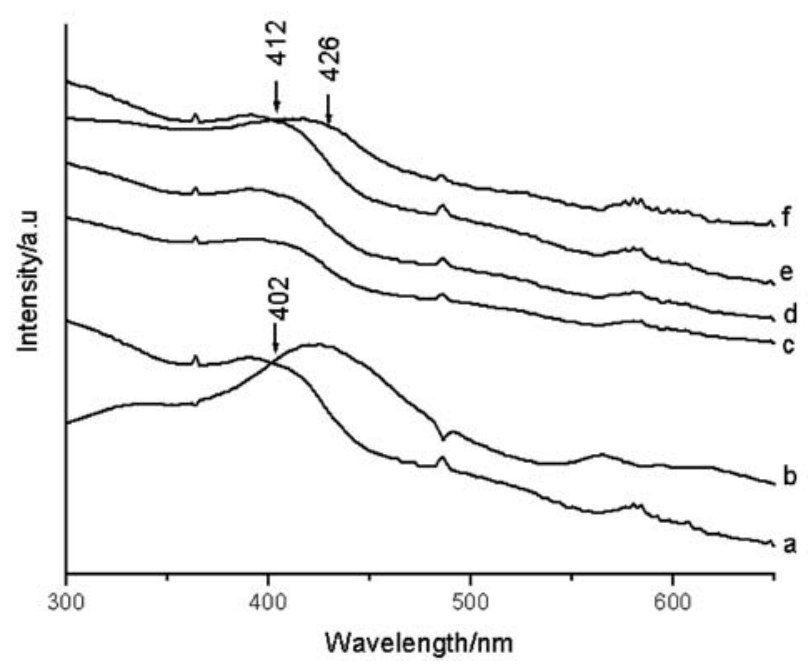

Figure 6. UV-Vis spectra (a) FePor 1-SGA; (b) FePor 1-SGB; (c) FePor 2-SGA; (d) FePor 2-SGB; (e) FePor 3-SGA; (f) FePor 3-SGB.

FePor dimeric species (e.g., $\mu$-oxo dimer species) are often observed when FePors are obtained in alkaline media, preferably in the presence of water traces. In this case, the FePor solution usually acquires a green color, and so does the solid obtained by immobilization of the dimeric species. The UV-Vis spectrum of the dimeric species in solution typically presents two bands, namely one in the $570-580 \mathrm{~nm}$ region (more intense) and another between $610-620 \mathrm{~nm}$ (shoulder). Furthermore, the Soret band shifts to lower energy, compared with the corresponding band for the monomeric species. ${ }^{41}$ Due to the difficult analysis of the visible region of the spectrum in the case of FePor immobilization in solid supports like silica, the presence of some dimeric species in the support cannot be discarded, mainly in the silica solids obtained by the base-catalyzed sol-gel process. However, if this dimerization process took place, it occurred in minor proportion, since the EPR spectra (Figure 4) indicate the presence of monomeric iron(III) porphyrin species. The $\mu$-oxo dimeric species of FePors are EPR silent, due to the antiferromagnetic coupling of the iron ions via $\mu$-oxo bridge..$^{41}$

From these results, we can assume that the FePor molecules were really introduced into the silica gels. ${ }^{21}$

Thermogravimetric and differential thermal analyses (TGA and DTA) (figure not shown) provide information on the thermal stability of the solids obtained after FePor immobilization. Such analyses give evidence of two events. Firstly, there is a mass loss of around 20\%, which is associated with an endothermic transition centered at $150{ }^{\circ} \mathrm{C}$ and corresponds to loss of adsorbed $\mathrm{H}_{2} \mathrm{O}$ molecules. There is another mass loss between 200 and
$600{ }^{\circ} \mathrm{C}$, attributed to FePor decomposition, followed by condensation and collapse of the inorganic structure, ${ }^{37}$ resulting in $\mathrm{SiO}_{2}$. FePor loss occurs approximately between 400 and $600^{\circ} \mathrm{C}$, and the residual groups are lost from 200 to $650{ }^{\circ} \mathrm{C}$. There is no exothermic effect. Similar results were obtained for all the FePor-SG solids.

The surface area and porosity of the materials, determined from nitrogen adsorption, are shown in Table 2. Comparison of the materials obtained by acid or basic catalysis reveals that the SGB materials have the largest surface areas. Indeed, low surface area values are found for materials obtained by acid catalysis.

Table 2. Physicochemical properties of FePor-SGA and FePor-SGB materials

\begin{tabular}{lccc}
\hline Sample & $S_{\mathrm{BET}}\left(\mathrm{m}^{2} \mathrm{~cm}^{-1}\right)^{\mathrm{a}}$ & $V_{\mathrm{p}}\left(\mathrm{cm}^{3} \mathrm{~g}^{-1}\right)^{\mathrm{a}}$ & $\mathrm{D}_{\mathrm{p}}(\AA)^{\mathrm{a}}$ \\
\hline FePor 1-SGB & 266 & 0.65 & 98.0 \\
FePor 3-SGB & 255 & 0.41 & 64.5 \\
FePor 2-SGA & 35 & 0.02 & 24.0 \\
FePor 3-SGA & 0.01 & -- & -- \\
\hline
\end{tabular}

${ }^{\text {aBET surface area }\left(S_{\mathrm{BET}}\right) \text {, average pore volume }\left(V_{\mathrm{p}}\right) \text { and average pore }}$ diameter $\left(D_{\mathrm{p}}\right)$ of the FePor-SGA/SGB samples.

As already mentioned, the $\mathrm{pH}$ employed in the preparation of the silica support influences the characteristics of the obtained material. A highly branched material is achieved by acid catalysis (SGA), which usually contains a large number of micropores. If the FePor is located on the surface of SGA, it hinders nitrogen gas adsorption by the FePor-SGA catalyst, thereby making this type of analysis impossible.

\section{Catalytic oxidation reactions}

The catalytic activities of FePors $\mathbf{1}, \mathbf{2}$ and $\mathbf{3}$ (homogeneous catalysis) and those of the corresponding supported catalysts (heterogeneous catalysis) in the oxidation of three substrates, namely two alkenes $((Z)$-cyclooctene and cyclohexene) and one alkane (cyclohexane), were investigated (Figure 7). The results are displayed in Tables 3 to 5 . The catalytic yields depicted in these tables can be attributed to all the solids resulting from immobilization of the FePs in the silicas (SGA or SGB), since control reactions carried out with the pure matrices (containing no FePor) did not furnish any oxidation products.

Since the first report of Groves et al. ${ }^{42}$ iodosylbenzene $(\mathrm{PhIO})$ has been frequently employed as oxygen transfer agent in oxidation reactions using metalloporphyrins. Those authors demonstrated that this reactant is relatively inert in the absence of the catalyst and reacts with FePors to produce the intermediate active species oxoiron(IV) 


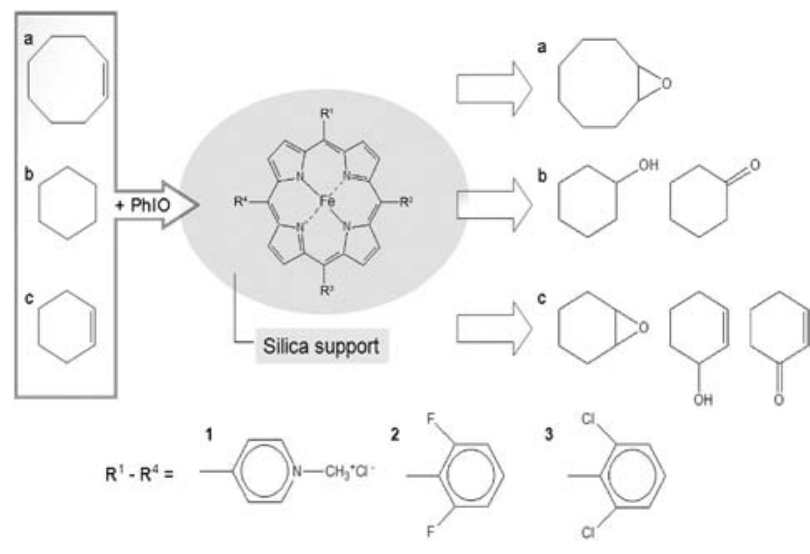

Figure 7. Schematic representation of the catalytic oxidation reactions using FePor-SG as heterogeneous catalysts.

porphyrin cation radical complex $\left(\mathrm{O}=\mathrm{Fe}^{\mathrm{IV}}\right.$ porphyrin $\left.{ }^{\circ+}\right)$. Furthermore, the polymeric nature of this oxidant is efficient to suppress metal-mediated free radical propagation reactions often observed in the case of cytochrome P-450 mimetic systems. ${ }^{43}$

A UV-Vis spectrum of the supernatant reaction solution was recorded after all the catalytic reactions. The typical Soret band of FePors was not detected in any of the cases, proving that no catalyst leaching form the support occurred in any of the reaction conditions investigated in this study. In other words, the catalysis is truly heterogeneous.

\section{(Z)-Cyclooctene}

It is well established that the oxidation of $(Z)$ cyclooctene by metalloporphyrin/PhIO systems produces epoxide as the sole oxidation product, with no traces of allylic alcohol or ketone. ${ }^{44}$ For this reason, this alkene is frequently employed as a diagnostic substrate in biomimetic catalytic systems involving metalloporphyrins. In this work, we used this substrate to investigate the efficiency and stability of the immobilized FePors as catalysts for alkene oxidation by PhIO. This study also provided information about the accessibility of the substrate and the oxidant to the iron(III) sites in the immobilized catalyst.

Table 3 shows the results from $(Z)$-cyclooctene oxidation with different catalysts. The reactions were accomplished in both homogeneous and heterogeneous environments.

The homogeneous FePors $\mathbf{2}$ and $\mathbf{3}$ furnish high epoxide yields (Table 3 , runs 4 and 7). FePor $\mathbf{1}$ is not completely solubilized in the reaction solvent, accounting for the lower yields (run 1). Both FePors $\mathbf{2}$ and $\mathbf{3}$ are expected to be highly efficient catalysts for oxidation reactions. This is because the electronegative substituents on their phenyl rings contribute to a reduction of the electronic
Table 3. (Z)-cyclooctene oxidation by PhIO catalyzed by FePors in homogeneous and heterogeneous media ${ }^{\mathrm{a}}$

\begin{tabular}{lccc}
\hline Catalyst $^{\mathrm{b}}$ & \multirow{2}{*}{ Run } & \multicolumn{2}{c}{ Epoxide yield $(\%)$} \\
\cline { 2 - 4 } & & $1 \mathrm{~h}^{\mathrm{c}}$ & $24 \mathrm{~h}^{\mathrm{d}}$ \\
\hline FePor 1 & 1 & 60 & 66 \\
FePor 1-SGA & 2 & 42 & 28 \\
FePor 1-SGB & 3 & 27 & 44 \\
FePor 2 & 4 & 65 & 79 \\
FePor 2-SGA & 5 & 86 & 97 \\
FePor 2-SGB & 6 & 60 & 69 \\
FePor 3 & 7 & 68 & 83 \\
FePor 3-SGA & 8 & 63 & 75 \\
FePor 3-SGB & 9 & 65 & 83 \\
SGA + PhIO (control) & 10 & 5 & - \\
SGB + PhIO (control) & 11 & 5 & - \\
PhIO (control) & 12 & 5 & - \\
\hline
\end{tabular}

${ }^{a}$ The yield of cyclooctene oxide was calculated on the basis of the amount of $\mathrm{PhIO}$ used in the reaction. The results represent an average of at least duplicate reactions. FePor/PhIO/(Z)-cyclooctene molar ratio = 1:50:5000. ${ }^{b}$ The piece of glass obtained from the sol-gel process was hardly grounded, and the resulting powder was used as catalyst. Reaction time: ${ }^{\mathrm{c}} 1$ and ${ }^{\mathrm{d}} 24 \mathrm{~h}$.

density on the porphyrin ring, thereby stabilizing the catalyst against oxidative degradation. ${ }^{45}$ Furthermore, the relative solubility observed for the three FePors in the reaction solvent (dichloromethane/acetonitrile mixture) is FePor $\mathbf{3}>$ FePor $\mathbf{2}>>$ FePor $\mathbf{1}$, and this relative solubility can also explain the catalytic yields depicted in Table 3.

All the immobilized FePors display catalytic activity for (Z)-cyclooctene oxidation, thus confirming that the immobilization process and the experimental conditions did not destroy or transform the FePors into inactive species (Table 3).

In reactions performed with the solid obtained by the sol-gel process (heterogeneous catalysis, Table 3 , runs 2 , $3,5,6,8$ and 9) the overall results obtained at one hour of reaction are generally lower than those obtained at the same reaction time with the corresponding homogeneous catalysts. It is frequently reported that the catalytic activity of metalloporphyrins is reduced upon immobilization, because the access of both the substrate and the oxidant to the active site is limited by the structure of the support. ${ }^{46}$ This seems to be the case with the heterogeneous catalysts investigated here, except for the FePor 3-SGA solid (Table 3, run 5), which furnished higher yields after FePor immobilization in SGA. Table 3 shows that better catalytic results are achieved at $24 \mathrm{~h}$. This suggests that access of the reactants to the metallic catalytic center is facilitated after longer contact time. FePor 1-SGA is an exception (Table 3, run 2), since longer reaction time actually decreased 
product yield. This might be related to the FePor structure, which is not robust enough to support long reaction times. Thus, some FePor destruction may have occurred.

Generally, the solids prepared by acid catalysis (FePorSGA) led to better catalytic yields (Table 3, runs 2 and 5) than those synthesized by the base-catalyzed sol-gel route (FePor-SGB) (Table 3, runs 3 and 6). These results agree with the characterization data obtained for the solids by EPR and UV-Vis spectroscopy, suggesting that some dimeric FePor species (e.g., $\mu$-oxo dimer species) could be present together with the monomer in SGB, prepared by the base-catalyzed sol-gel process. The dimerization of FePor $\mathbf{1}$ and FePor $\mathbf{2}$ can take place because both porphyrin ligands do not have bulky substituents like FePor $\mathbf{3}$, which contains chlorine atoms that protect the porphyrin ring from dimerization. In fact, some dimeric and other aggregated species have been well characterized for FePors $\mathbf{1}^{47-49}$ and 2. Moreover, dimeric FePor $\mathbf{1}$ presents low catalytic yield compared with the monomeric species. ${ }^{50}$

\section{Cyclohexane}

In general, the oxidation of cyclohexane with iodosylbenzene in the presence of metalloporphyrins commonly yields cyclohexanol and cyclohexanone as major products, so this kind of catalyst is frequently considered cytochrome P-450 biomimetic models. High selectivity for the alcohol product is also observed. Alcohol formation occurs via proton abstraction by the active intermediate species oxoiron(IV) porphyrin $\pi$-cation radical complex, responsible for the hydroxylation reaction. ${ }^{51}$ Cyclohexane is a very useful substrate to investigate the efficiency of FePors as catalysts for alkane hydroxylation by iodosylbenzene. The activation of inert $\mathrm{C}-\mathrm{H}$ bonds in alkanes calls for more drastic conditions than those necessary for alkene functionalization, thus allowing for a better differentiation between the performances of a metalloporphyrin catalyst in solution and immobilized in a solid support. ${ }^{10,42,48,52,53}$

Table 4 shows that FePors $\mathbf{1}, \mathbf{2}$ and $\mathbf{3}$ were catalytically active for cyclohexane oxidation (homogeneous catalysis). However, we had expected that both FePors $\mathbf{2}$ and $\mathbf{3}$ would present higher efficiency than FePor $\mathbf{1}$ (Table 4, run 1), since the former contain robust, electron-deficient porphyrin structures. In fact, high product yields (59\%) and high selectivity for cyclohexanol were observed for FePor 2 (Table 4, run 4), but not so high for FePor 3 (Table 4, run $7,37 \%$ ). The relative solubility and electron-deficiency caused by the electron-withdrawing substituents on the porphyrin ring of both FePors $\mathbf{2}$ and $\mathbf{3}$ could explain these results. Nam et al. observed that the oxidizing power
Table 4. Cyclohexane oxidation by PhIO catalyzed by FePors in homogeneous and heterogeneous media ${ }^{a}$

\begin{tabular}{lccc}
\hline Catalyst $^{\mathrm{b}}$ & Run & $\begin{array}{c}\text { Alcohol yield } \\
(\%)^{\mathrm{c}}\end{array}$ & $\begin{array}{c}\text { Ketone yield } \\
(\%)^{\mathrm{d}}\end{array}$ \\
\hline FePor 1 & 1 & 6 & 1 \\
FePor 1-SGA & 2 & 7 & - \\
FePor 1-SGB & 3 & 2 & 1 \\
FePor 2 & 4 & 55 & 1 \\
FePor 2-SGA & 5 & 4 & - \\
FePor 2-SGB & 6 & 3 & 14 \\
FePor 3 & 7 & 37 & 2 \\
FePor 3-SGA & 8 & 2 & 20 \\
FePor 3-SGB & 9 & 7 & 7 \\
SGA + PhIO (control) & 10 & trace & - \\
SGB + PhIO (control) & 11 & trace & - \\
PhIO (control) & 12 & trace & - \\
\hline
\end{tabular}

${ }^{\mathrm{a}}$ The yields of the reactions were calculated on the basis of the amount of PhIO used in the reaction. The results represent an average of at least duplicate reactions. FePor/PhIO/cyclohexane molar ratio = 1:50:5000. ${ }^{\mathrm{b}}$ The piece of glass obtained from the sol-gel process was hardly grounded, and the resulting powder was used as catalyst. ${ }^{\circ}$ Cyclohexanol and ${ }^{\mathrm{d} C y c l o h e x a n o n e . ~}$

of the catalytic intermediate active species oxoiron(IV) porphyrin $\pi$-cation radical complex is directly related to the electronic nature of the porphyrin ligand. ${ }^{54}$ Probably, the electron-deficiency of the porphyrin ligands containing chlorine and fluorine substituents is very similar, but any difference in their oxidizing capacity is more evident when the substrate is hard to oxidize, as in the case of cyclohexane and other alkanes. ${ }^{55}$

In line with the results from $(Z)$-cyclooctene oxidation (Table 3), immobilization of all three FePors rendered solids with low catalytic capacity for selective cyclohexane hydroxylation as well as lower alcohol yield, compared with the homogeneous counterparts. For the less reactive cyclohexane, access to the catalytic centre could be even more critical than in the case of highly reactive substrates like (Z)-cyclooctene. ${ }^{38}$

In contrast with homogeneous media, reactions 6, 8 and 9 (Table 4) reveal large ketone production. This behavior is a consequence of the proposed reaction mechanism. ${ }^{51}$ For homogeneous catalytic systems using FePors, Lindsay-Smith et al..$^{56}$ observed that, depending on the porphyrin structure and catalysis conditions, ketone was formed from further alcohol oxidation. As for the heterogeneous catalysis investigated in the present work, the pores of the silica structure can delay diffusion of the alcohol into the solution, and the presence of this product near the active metal centre can result in its further oxidation, in a competitive process with cyclohexane. This situation is more dramatic when the structure of the 
porphyrin ring contains bulkier substituents, as in the case of FePor 3 (Table 4, run 2). In fact, we recently observed the same catalytic behavior when metalloporphyrins immobilized in LDH (layered double hydroxide) ${ }^{55}$ and silica ${ }^{19}$ were employed as catalysts for alkane oxidation. Catalytic experiments at different reaction times corroborated ketone production from alcohol re-oxidation.

\section{Cyclohexene}

The products generated from cyclohexene oxidation mediated by $\mathrm{PhIO} / \mathrm{FePor}$ systems result from a competition between the $\mathrm{C}=\mathrm{C}$ and allylic $\mathrm{C}-\mathrm{H}$ groups on the alkene for the electrophilic active species oxoiron(IV) porphyrin $\pi$-cation radical complex ${ }^{57,58}$ formed in the reaction between FePor and iodosylbenzene. Oxidation of these groups should lead to cyclohexene oxide and/or allylic alcohol (1-cyclohexen-3-ol) and ketone (1-cyclohexen-3one), respectively. ${ }^{59,60}$ Homogeneous $\mathrm{PhIO} / \mathrm{FePor}$ systems furnish allylic products in minor yields compared with the epoxide, and the efficiency and selectivity of the catalytic reaction toward the latter compound is controlled by the reaction conditions (solvent, temperature, inert atmosphere and reactants molar ratio), ${ }^{61}$ the structure of the porphyrin ring, and the presence of axial ligands to the iron center. ${ }^{35,36}$

In a preliminary examination (Table 5), the use of the three investigated FePors in homogeneous and heterogeneous systems for cyclohexene oxidation gave epoxide and significant yields of allylic oxidation products (Table 5). However, the control reactions indicate that the silica (SGA and SGB) support alone largely contributes to the total product yields (Table 5, runs 10 and 11), and the reaction performed with $\mathrm{PhIO}$ and substrate only (Table 5, run 12) also leads to significant amounts of allylic products. Therefore, the results concerning the final percentage of product yields obtained after deduction of the yields achieved in the control reactions should provide a more realistic picture of the catalytic results obtained with both homogeneous and heterogeneous systems.

The product distributions and yields achieved in the oxidation reactions catalyzed by the FePors are consistent with the presence of dioxygen in the reaction medium, especially in the case of the heterogeneous systems. Controlling the presence of dioxygen is a problem inherent to heterogeneous catalysis since this gas might be present in the voids of the small pores and cavities of the silica, making it difficult to ensure its absence. The presence of dioxygen in the support was confirmed by the high yields of allylic products obtained when only the SGA or SGB support (with no immobilized FePor) was used as catalyst (Table 5, runs 10 and 11).
Table 5. Cyclohexene oxidation by PhIO catalyzed by FePors in homogeneous and heterogeneous media ${ }^{\mathrm{a}}$

\begin{tabular}{lccc}
\hline Catalyst & Run & $\begin{array}{c}\text { Epoxide yield } \\
(\%)^{\mathrm{b}}\end{array}$ & $\begin{array}{c}\text { Ketone + Alcohol } \\
(\%)^{\mathrm{c}}\end{array}$ \\
\hline FePor 1 & 1 & 36 & 40 \\
FePor 1-SGA & 2 & 16 & $>100$ \\
FePor 1-SGB & 3 & 26 & $>100$ \\
FePor 2 & 4 & 13 & 36 \\
FePor 2-SGA & 5 & 34 & 22 \\
FePor 2-SGB & 6 & 57 & $>100$ \\
FePor 3 & 7 & 35 & 42 \\
FePor 3-SGA & 8 & 21 & $>100$ \\
FePor 3-SGB & 9 & 72 & $>100$ \\
SGA + PhIO (control) & 10 & 6 & $>100$ \\
SGB + PhIO (control) & 11 & 6 & $>100$ \\
PhIO (control) & 12 & - & 25 \\
\hline
\end{tabular}

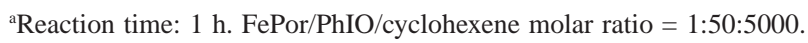
The yield of the reaction was calculated on basis of the amount of $\mathrm{PhIO}$ employed used in the reaction. The results represent an average of at least duplicate reactions. All the catalyst solids used were macerated. ${ }^{\mathrm{b}}$ Epoxide $=$ cyclohexene oxide. ${ }^{\mathrm{c}} \mathrm{Ketone}+\mathrm{Alcohol}=2$-cyclohexen-1-one + 2-cyclohexen-1-ol.

In fact, we recently reported that the oxidation of cyclohexene in the presence of immobilized FePor and air, under magnetic stirring, in catalytic reaction conditions similar to those employed here, resulted in the preferable conversion of cyclohexene to allylic products. ${ }^{35}$ This result was consistent with a free-radical autooxidation mechanism mediated by the FePor in solution or immobilized into $\mathrm{Zn}_{\mathrm{n}} \mathrm{Al}-\mathrm{LDH} .{ }^{62}$ Most of the allylic products from cyclohexene conversion are produced when the substrate and solvent are stirred magnetically in air, for the same time period and under the same temperature conditions as the catalytic reaction.

In spite of the fact that the allylic products yields were superior to $100 \%$ because of the uncontrolled presence of oxygen from air, the catalyst FePor 2-SGB (Table 5, run 9) furnished high epoxide yield, showing that this system can be very efficient for cyclohexene oxidation.

The recyclability of the solids resulting from immobilization of the three FePors was investigated in the oxidation of cyclohexene. It was done in the same experimental conditions employed during the first use of each catalyst, after the solids had been recovered and washed for removal of traces of products (or iodobenzene and iodosylbenzene) that could have remained inside the silica structure. UV-Vis analysis of the solvents used in the washing process proved that there was no FePor leaching from the support. The catalyst solids were reused in two further catalytic reactions and, in spite of the high quantity 
of allylic products, the epoxide yields were similar to those achieved during the first use of the catalyst.

Surprisingly, despite the low amount of FePor in the silica, there is a non-linear dependence of catalytic efficiency on surface area and loading. Additionally, there is no direct relationship between catalytic efficiency and surface area or FePor loading on the support for any of the studied substrates. Surface area depends solely on the silica preparation method (acid or basic catalysis).

Based on the catalytic results and characterization of the solids, we argue that catalyst efficiency is more related to FePor dispersion on the support than to the final surface area of the catalyst. This dispersion can be affected by such parameters as FePor interaction with the support, which in turn is governed by the $\mathrm{pH}$ used during silica preparation. Strong interactions between the catalyst and the support may lead to better FePor dispersion in the matrix.

Finally, it is noteworthy that the catalysts prepared here have lower FePor loadings compared with other systems reported in the literature. Nonetheless, our catalysts led to similar (Z)-cyclooctene conversion results. For example, Battioni et al ${ }^{63}$ reported that the FePor-supported silica system catalyzed the epoxidation of cyclooctene by $\mathrm{PhIO}$ with high epoxide yields (> 90\%), by employing materials with high surface area $\left(c a .700 \mathrm{~m}^{2} \mathrm{~g}^{-1}\right)$ and catalyst loading of $150 \mathrm{mg} \mathrm{g}^{-1}$ support. The catalytic yields are similar to those achieved with the SGA-FePor or SGB-FePor catalysts prepared by us, but it may be considered that the catalyst prepared by Battioni et al..$^{63}$ has an iron porphyrin content of $15 \mathrm{~g} \mathrm{~g}^{-1}(15 \%)$, which is 10 times higher than the content in our SG-FePor catalysts (Table 1, 0.8 to $2.1 \mathrm{~g} \mathrm{~g}^{-1}$ ).

\section{Conclusions}

Three structurally different FePors have been successfully immobilized in silica prepared by means of an inexpensive, practical experimental procedure using the sol-gel process. Factors such as the nature of the FePor and the type of catalyst used during silica synthesis influence the morphology of the obtained particles. Charged FePors such as [Fe(TMPyP)] furnish hollow spherical particles. Alternatively, the FePor may direct the construction of hollow spheres through a facile synthesis carried out in mild conditions, without the need for templates or the use of emulsifying methods. In general, the immobilization process does not seem to favor the catalytic activity of the FePors investigated here. Probably, the access of the oxidant and substrate to the active site of the immobilized FePor is difficult, and the catalytic yield for the heterogeneous catalyst is lower than that achieved with the parent homogeneous system. [Fe(TDFPP)] is an exception, since it led to higher yields for ( $Z$ )-cyclooctene and cyclohexene oxidation compared with the corresponding homogeneous system. Nevertheless, it must be borne in mind that FePor heterogenization is advantageous since it enables catalyst recycling, thereby resulting in high total turnovers.

\section{Acknowledgments}

The authors are grateful to Conselho Nacional de Desenvolvimento Científico e Tecnológico (CNPq), Coordenação de Aperfeiçoamento de Pessoal de Nível Superior (CAPES), Fundação Araucária, Fundação da Universidade Federal do Paraná (FUNPAR) and Universidade Federal do Paraná (UFPR) for financial support. They also thank the Centro de Microscopia Eletrônica da UFPR for the TEM analyses.

\section{References}

1. Kosal, M. E.; Suslick, K. S.; J. Solid. State Chem. 2000, 152, 87.

2. Costa, C. O.; Souza, A. A.; Luz, R. C. S.; Lemos, T. L. G.; Pessoa, O. D. L.; Kubota L. T.; Goulart M. O. F.; J. Braz. Chem. Soc. 2008, 19, 697.

3. Silva, A. R.; Pelegrino, A. C.; Tedesco, A. C.; Jorge, R. A.; J. Braz. Chem. Soc. 2008, 19, 491.

4. Nakagaki, S.; Castro, K. A. D. F.; Machado, G. S.; Halma, M.; Drechsel, S. M.; Wypych, F.; J. Braz. Chem. Soc. 2006, 17, 1672.

5. Suslick, K. S.; Bhyrappa, P.; Chou, J.-H.; Kosal, M. E.; Nakagaki, S.; Smithenry, D. W.; Wilson, S. R.; Acc. Chem. Res. 2005, 38, 283.

6. Benedito, F. L.; Nakagaki, S.; Saczk, A. A.; Peralta-Zamora, P. G.; Costa, M. C. M.; Appl. Catal., A 2003, 250, 1.

7. Iamamoto, Y.; Idemori, Y. M.; Nakagaki, S.; J. Mol. Catal. A: Chem. 1985, 99, 187.

8. Nakagaki, S.; Xavier, C. R.; Wosniak, A. J.; Mangrich, A. S.; Wypych, F.; Cantão, M. P.; Denicoló, I.; Kubota, L. T.; Colloids Surf., A 2000, 168, 261.

9. Herron, N.; J. Coord. Chem. Rev. 1998, 19, 25.

10. Barloy, L.; Lallier, J. P.; Battioni, P.; Mansuy, D.; Pitfard, Y.; Tournoux, M.; Valim, J. B.; Jones, W.; New J. Chem. 1992, 16, 71.

11. Machado, A. M.; Wypych, F.; Drechsel, S. M.; Nakagaki, S.; J. Colloid Interface Sci. 2002, 254, 158.

12. Faria, A. L.; Mac Leod, T. O. C.; Barros, V. P.; Assis, M. D.; J. Braz. Chem. Soc. 2009, $20,895$.

13. Nakagaki, S.; Halma, M.; Bail, A.; Arízaga, G. G. C.; Wypych, F.; J. Colloid Interface Sci. 2005, 281, 417.

14. Wypych, F.; Bail, A.; Halma, M.; Nakagaki, S.; J. Catal. 2005, $234,431$. 
15. Nakagaki, S.; Wypych, F.; J. Colloid Interface Sci. 2007, 315, 145.

16. Vidoto, E. A.; Moreira, M. S. M.; Vinhado, F. S.; Ciuffi, K. J.; Nascimento, O. R.; Iamamoto, Y.; J. Non-Cryst. Solids 2002, 304, 151.

17. Sacco, H. C.; Ciuffi, K. J; Biazzotto, J. C.; Zuccki, M. R.; Leite, C. A. M.; Nascimento, O. R.; Serra, O. A.; Iamamoto, Y.; J. Non-Cryst. Solids 2000, 273,150.

18. Oliveira, D. C.; Sacco, H. C.; Nascimento, O. R.; Iamamoto, Y.; Ciuffi, K. J.; J. Non-Cryst. Solids 2001, 284, 28.

19. Papacídero, A. T.; Rocha, L. A.; Caetano, B. L.; Molina, E.; Sacco, H. C.; Nassar, E. J.; Martinelli, Y.; Mello, C.; Nakagaki, S.; Ciuffi, K. J.; Colloids Surf., A 2006, 275, 27.

20. Podbielska, H.; Ulatowska-Jarza, A.; Müller, G.; Holowacz, I.; Bauer, J.; Bindig, U.; Biom. Eng. 2007, 24, 425.

21. Tanaka, H.; Yamada, T.; Sugiyama, S.; Shiratori, H.; Hino, R.; J. Colloid Interface Sci. 2005, 286, 812.

22. Dror, I; Baram, D.; Berkowitz, B.; Environ. Sci. Technol. 2005; 39, 1283.

23. Wang, H.; Song, Y.; Wang, Z.; Medforth, C. J.; Miller, J. E.; Evans, L.; Li, P.; Shelnutt, J. A.; Chem. Mat. 2008, 20, 7434.

24. Halma, M.; Wypych, F.; Dreschel, S. M.; Nakagaki, S.; J. Porph. Phth. 2002, 6, 502.

25. Stöber, W.; Fink, A.; J. Colloid Interface Sci. 1968, 62, 26.

26. Sharefkin, J. G.; Saltzmann, H.; Org. Synth. 1963, 43, 62.

27. Lucas, J.; Kennedy, E. R.; Forno, M. W.; Org. Synth. 1963, 43, 483.

28. Rocha-Gonsalves, A. M.; J. Heterocycl. Chem. 1991, 28.

29. Smith, K. M.; Porphyrins and Metalloporphyrins, Elsevier: Amsterdam, 1975, p. 317.

30. Adler, A.; Longo, F. R.; J. Am. Chem. Soc. 1964, 86, 3145.

31. Adler, A.; Longo, F. R.; Kampas, F.; Kim, J.; Inorg. Nucl. Chem. 1979, 32, 2443.

32. Buckley, A. M.; Greenblatt, M.; J. Chem. Educ. 1994, 71, 599.

33. Brunauer, S.; Emmett, P. H.; Teller, E.; J. Am. Chem. Soc. 1938, $60,309$.

34. Brinker, C. J.; Scherer, G. W.; Sol-Gel Science. The Physics and Chemistry of Sol-Gel Processing, Academic Press: San Diego, 1990, ch. 3.

35. Halma, M.; Castro, K. A. D. F.; Taviot-Gueho, C.; Prévot, V.; Forano, C.; Wypych, F.; Nakagaki, S.; J. Catal. 2008, 25, 233.

36. Nakagaki, S.; Benedito, F. L.; Wypych, F.; J. Mol. Catal. A: Chem. 2004, 217, 121.

37. Halma, M.; Bail, A.; Wypych, F.; Nakagaki, S.; J. Mol. Catal. A: Chem. 2006, 243, 44.

38. Piovezan, C.; Castro, K. A. D. F.; Drechsel, S. M.; Nakagaki, S.; Appl. Catal., A 2005, 293, 97.

39. Trytek, M.; Fiedurek, J.; Lipke, A.; Radzki, S.; J. Sol-Gel Sci. Technol. 2009, 51, 272.

40. Rabelo, S. L. H.; Conçalves, A. R.; Pereira, M. M.; Simões, M. M. Q.; Neves, M. G. P. M. S.; Cavaleiro, J. A. S.; J. Mol. Catal. A: Chem. 2006, 256, 221.
41. Manso, C. M. C. P.; Neri, C. R.; Vidoto, E. A.; Sacco, H. C.; Ciuffi, K. J.; Iwamoto, L. S.; Iamamoto, Y.; Nascimento, O. R.; Serra, O. A.; J. Inorg. Biochem. 1999, 73, 85.

42. Groves, J. T.; Nemo, T. E.; Myers, R. S.; J. Am. Chem. Soc. 1979, 101, 1032.

43. Groves, J. T.; J. Inorg. Biochem. 2006, 100, 434.

44. Appleton, A. J.; Evans, S.; Lindsay-Smith, J. R.; J. Chem. Soc., Perkin Trans. 2. 1996, 281.

45. Dolphin, D.; Traylor, T. G.; Xie, L. Y.; Acc. Chem. Res. 1997, $30,251$.

46. Nakagaki, S.; Machado, G. S.; Halma, M.; Marangon, A. A. S.; Castro, K. A. D. F.; Mattoso, N.; Wypych, F.; J. Catal. 2006, 242, 110.

47. Harris, F. L.; Toppen, D. L.; Inorg. Chem. 1978, 17, 71.

48. Yamamoto, Y.; Idemori, I. M.; Nakagaki, S.; J. Mol. Catal. A: Chem, 1995, 99, 187.

49. Miskelly, G. M.; Webley, W. S.; Clark, C. R.; Buckingham, D. A.; Inorg. Chem. 1988, 27, 3773.

50. Nakagaki, S.; Iamamoto, Y.; Baffa, O.; Nascimento, O. R.; Inorg. Chim. Acta 1991, 186, 39.

51. Groves, J. T.; Nemo, T. E.; J. Am. Chem. Soc. 1983, 105, 6243.

52. Groves, J. T.; Haushalter, R. C.; Nakamura, M.; Nemo, T. E.; Evans, B. J.; J. Am. Chem. Soc. 1981, 103, 2884.

53. Berkowitz, J.; Ellison, G. B.; Gutman, D.; J. Phys. Chem. 1994, 98, 2744.

54. Goh, Y. M.; Nam, W.; Inorg. Chem. 1999, 38, 914.

55. Halma, M.; Castro, K. A. D. F.; Prévot, V.; Forano, C.; Wypych, F.; Nakagaki, S.; J. Mol. Catal. A: Chem. 2009, 310, 42.

56. Inchley, P.; Lindsay-Smith, J. R.; Lower, R. J.; New J. Chem. 1989, 13, 669.

57. Groves, J. T.; Subramanian, D. R.; J. Am. Chem. Soc. 1984, 106, 2177.

58. Lindsay-Smith, J. R.; Sleath, P. R.; J. Chem. Soc., Perkin Trans. 2 1982, 1009.

59. Groves, J. T.; Nemo, T. E.; J. Am. Chem. Soc. 1983, 105, 5786.

60. Appleton, A. J.; Evans, S.; Lindsay-Smith, J. R.; J. Chem. Soc., Perkin Trans. 2 1995, 28.

61. Song, W. J.; Ryu, Y. O.; Song, R.; Nam, W.; J. Biol. Inorg. Chem. 2005, 10, 294.

62. Paulson, D.; Ullman, R.; Sloane, R. B.; J. Chem. Soc., Chem. Commun. 1984, 186.

63. Battioni, P.; Cardin, E.; Louloudi, M.; Schollhorn, B.; Spyroulias, G. A.; Mansuy, D.; TrayIor, T. G.; Chem. Commun. 1996, 2037.

Received: September 28, 2009 Web Release Date: April 22, 2010

FAPESP helped in meeting the publication costs of this article. 